\title{
SERVICE DOMINANT LOGIC - AN EXAMPLE OF COMPETITIVE ADVANTAGE
}

\author{
Kenneth Le Meunier-FitzHugh, University of East Anglia. UK \\ Leslie Le Meunier-FitzHugh, University of East Anglia. UK \\ Roger Palmer, University of Bournemouth. UK \\ Moria Clark, Henley Business School, UK \\ Neil Hair, Rochester Institute of Technology, USA
}

\begin{abstract}
Value and the concept of value co-creation in marketing networks are growing in importance in providing competitive advantage in increasingly saturated markets. Service-Dominant logic (S-D logic) revolves around the concept that value is always co-created by customers and organizations together (Gronroos, 2006; Vargo and Lusch, 2004; 2008). This study provides a conceptual framework around the topic of co-creation, and identifies customer value creating processes, supplier value creating processes and encounter processes within a single case study of a pharmaceutical organization (called PharmCo for this study). This organization has successfully created its own competitive space, achieving $34 \%$ market growth, year-on-year, over a seven-year period. The paper summarizes some of the key conditions of service dominant logic (Ballantyne \& Varey, 2007), including value in use, stakeholder interaction, co-creation and the role of integrated networks, the role of the marketer as managing communication, interaction and relationships, between networks of co-operators.

Organizations with a service-dominated culture will create networks of supporting suppliers thereby enabling stronger solutions to be offered to customers (Sheth and Sharma, 2008). Value is always determined by the customer and co-created in the joint interaction (service) between buyer and seller (Vargo and Lusch, 2004). Co-creation of value is not simply inviting the customer to take part in the product development, but actually integrates the organization's offering into the lives of their customers (Payne et al., 2008). Co-creation at PharmCo took place in the 'disease space', within the network of relationships surrounding the patient, aimed at supporting the patient. Culture has been identified as a management opportunity to optimize human resources to improve business performance and is an attribute that is not easy to copy (Ogbonna and Harris, 2002). Culture may be employed to develop appropriate behaviors and attitudes in employees by focusing them on a set of meaningful and unified objectives (Chatman and Cha, 2003) leading to competitive advantage. The staff at PharmCo were encouraged to carry a small credit card-sized checklist summarizing what the organization's values and paradigm, including the purpose of 'providing life-transforming treatment'.
\end{abstract}

PharmCo realized that product alone would not solve the patient's problems and to help them beat their addition they created a network of expertise that the patient and their medical practitioners could access through a communications website. Exchange networks, typified by the interaction model and the role of actors, processes, bonds and resources, may be developed with external agencies and become part of organizational/marketing activities in service-dominated organizations (Lian and Laing, 2007). These networks may rely on a range of variables, e.g. social, power, diffusion, economic, and knowledge management, but all are based in communication ties (Katz and Lazer, 2002). PharmCo set up a value creation network, which is comprised of at least 36 distinct entities. Value was jointly created as members of the network, which is an organic and open-ended entity, because it grows and develops as the disease space evolves, delivered higher levels of service to the patient. The members of the network understanding the reason why they participate in the network, and underpins this with an understanding of how their interaction can support the patient to everyone's advantage.

The importance of the culture and the guiding principle of the focus to patient treatment have been emphasized, rather than the more direct commercial emphasis on selling products. This is very much a direct contrast to the conventional pharmaceutical business model, which involves large sales forces calling on physicians in order to "detail" the benefits of their particular medication and to encourage sales. Not only have PharmCo developed a very different approach in the context of the pharmaceutical industry they have also created a unique competitive advantage, which has been achieved through: The building of relationships through dialogue to create a network that is sustained by a common vision. Managers focused on providing comprehensive solutions to customers' needs, and competing in the "problem space" rather than the "marketplace". Marketing collateral is being used to resource the network, measure and provides the route to continual improvement. Network members understanding why and how they create and deliver value, and a relentless focus to providing customer solutions create the vision for the organization.

References Available Upon Request 